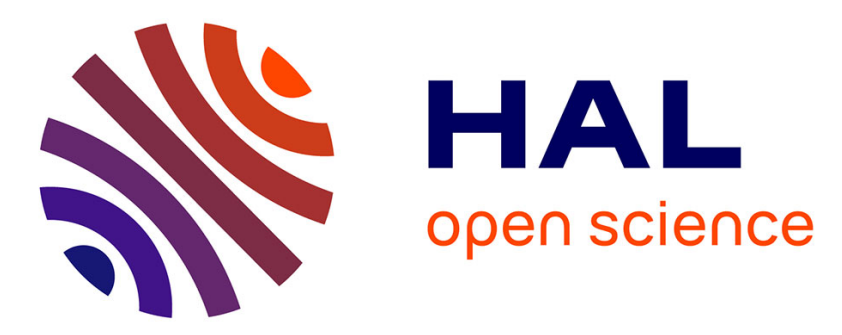

\title{
Characterization of the velocity field organization in heterogeneous media by conditional correlation
}

Tanguy Le Borgne, Jean-Raynald de Dreuzy, Philippe Davy, Olivier Bour

\section{To cite this version:}

Tanguy Le Borgne, Jean-Raynald de Dreuzy, Philippe Davy, Olivier Bour. Characterization of the velocity field organization in heterogeneous media by conditional correlation. Water Resources Research, 2007, 43 (2), pp.W02419. 10.1029/2006WR004875 . hal-00138043

\section{HAL Id: hal-00138043 https://hal.science/hal-00138043}

Submitted on 4 Feb 2016

HAL is a multi-disciplinary open access archive for the deposit and dissemination of scientific research documents, whether they are published or not. The documents may come from teaching and research institutions in France or abroad, or from public or private research centers.
L'archive ouverte pluridisciplinaire HAL, est destinée au dépôt et à la diffusion de documents scientifiques de niveau recherche, publiés ou non, émanant des établissements d'enseignement et de recherche français ou étrangers, des laboratoires publics ou privés. 


\title{
Characterization of the velocity field organization in heterogeneous media by conditional correlation
}

\author{
T. Le Borgne, ${ }^{1}$ J.-R. de Dreuzy, ${ }^{1}$ P. Davy, ${ }^{1}$ and O. Bour ${ }^{1}$ \\ Received 10 January 2006; revised 25 August 2006; accepted 18 September 2006; published 20 February 2007.
}

[1] The purpose of the present work is to quantify the correlation structure of simulated velocity fields in heterogeneous permeability fields and to discuss how to represent it in upscaled transport models. We investigate the velocity field correlation structure for multinormal log permeability fields. The simulated velocity distributions are analyzed in a Lagrangian framework, i.e., along the particles' paths. To quantify the different spatial organization of low- and high-velocity zones, we condition the estimated velocity correlation length and time on the initial particle velocity. The velocity correlation length is found to increase with the initial particle velocity, following a power law. Such an effect is likely due to the channeling of high-velocity zones, which implies that particles keep memory of their initial velocity over longer distances for high initial velocities than for low initial velocities. Two distinct regimes are identified for the velocity correlation time. For low initial particle velocity the correlation time is controlled by the large time needed to escape from the low-velocity zones. For high initial particle velocity it is controlled by the large time needed for particles to sample the whole velocity field, in particular low-velocity zones. One of the consequences of these results is that for such velocity fields the nonlinear dependence of both the correlation length and time on the particle initial velocity restricts the use of spatial or temporal Markovian assumptions for modeling velocity transitions in effective transport models.

Citation: Le Borgne, T., J.-R. de Dreuzy, P. Davy, and O. Bour (2007), Characterization of the velocity field organization in heterogeneous media by conditional correlation, Water Resour. Res., 43, W02419, doi:10.1029/2006WR004875.

\section{Introduction}

[2] Solute transport in aquifers is controlled by diffusion, local-scale dispersion, and spreading due to large-scale heterogeneity of the velocity field producing fast and slow paths. All together those processes produce the apparent large-scale dispersion. Predicting the influence of the geological heterogeneity on the solute mixing and spreading is required in many field applications (i.e., contaminant monitoring and remediation). For the case of moderately heterogeneous media, dispersion may be adequately predicted by the macrodispersion theory derived from first-order developments [Sudicky, 1986; Dagan, 1989; Gelhar, 1993]. However, an increasing number of field observations indicate that the Fickian model is not applicable to highly heterogeneous aquifers, which represent a large number of geological settings [Adams and Gelhar, 1992; Tsang and Neretnieks, 1998; Haggerty et al., 2000; Harvey and Gorelick, 2000; LaBolle and Fogg, 2001; Becker and Shapiro, 2003]. To account for that, several alternative transport models have been proposed [Berkowitz and Scher, 1998; Haggerty and Gorelick, 1995; Benson et al., 2000; Berkowitz et al., 2002; Becker and Shapiro, 2003]. These different models may correspond to different types of velocity field organization occurring in heterogeneous media. The important character-

\footnotetext{
${ }^{1}$ Géosciences Rennes, UMR 6118, Centre National de la Recherche Scientifique, Université de Rennes 1, Rennes, France.
}

Copyright 2007 by the American Geophysical Union. 0043-1397/07/2006WR004875 istics of the velocity field are the distribution of point values and the correlation structure, which quantifies the spatial organization of the velocity field. However, the relationship between the velocity field structure and the upscaled transport models is currently not clearly defined. The motivation of the present work is to propose a method to quantify the correlation structure of simulated velocity fields in heterogeneous permeability fields and to discuss how to represent it in upscaled transport models.

[3] Most of the past studies that quantify the velocity field correlation are based on the classical velocity field covariance [e.g., Salandin and Fiorotto, 1998]. However, such measure of spatial correlation does not resolve differences between high- and low-velocity zones' spatial organization [Zinn and Harvey, 2003]. Flow channeling and the presence of preferential high-velocity flow paths is often cited as the main reason for the observed non-Fickian transport effects [Tsang and Neretnieks, 1998]. Such qualitative observation implies that the spatial organization of low- and high-velocity zones is different. The presence of large velocity channels for instance may imply long-range velocity correlation for solute particles transported in large velocity zones. The objective of this paper is to improve the characterization of the velocity field organization by integrating information on the velocity magnitude. To do so, we analyze the Lagrangian velocity field (i.e., the distribution of velocity along the path of solute particles) obtained by particle tracking simulations in multinormal log permeability fields. 
[4] The velocity field correlation quantifies how a particle keeps memory of its initial particle velocity $v_{0}$. At early traveltimes or travel distance, the probability for the particle to move with the initial velocity $v_{0}$ is high. Hence the distribution of possible velocities, given than the particle started initially from the velocity $v_{0}$ is narrow and centered on $v_{0}$. At large traveltimes or distances, the probability distribution of possible velocities is the same whatever the initial velocities: the conditional velocity distributions reach an asymptotic velocity distribution. Thus the study of the evolution with traveltime or distance of the conditional velocity distributions allows to quantify the velocity field correlation function, conditioned on the initial velocity $v_{0}$.

\section{Numerical Procedure for Simulating Flow and Transport}

[5] Continuous heterogeneous media are classically modeled by lognormal correlated permeability fields. Several alternative representations of the permeability spatial variability in numerical models have been proposed. In particular, some representations include preferentially connected high-permeability zones [Gomez-Hernandez and Wen, 1998, Zinn and Harvey, 2003; Liu et al., 2004; Knudby and Carrera, 2005]. These representations allow investigating specific types of medium such as long-range correlated systems. In this study, we do not integrate any correlation more specific than a classical exponential correlation function in the permeability field model. Our aim is (1) to investigate how a nonmultinormal velocity field structure may arise in multinormal log permeability fields and (2) to propose a general methodology for the analysis of the velocity field correlation structure that may be applied to different types of permeability fields.

[6] The lognormal permeability distribution is parameterized by its lognormal mean $\langle\ln K\rangle$, its variance $\sigma_{\ln K}^{2}$ and a correlation length $\lambda_{\operatorname{lnK}}$. We investigate systems where $\log$ permeability field variance, $\sigma_{\operatorname{lnK}}^{2}$, ranges from 0.25 to 3 covering a large variety of natural aquifers [Gelhar, 1993]. Spatially correlated multinormal log conductivity fields are generated using a fast Fourier transform [Ruan and McLaughin, 1998] (Figure 1a). We restrict our study to two-dimensional systems. Flow is induced by a mean head gradient $\nabla h$. Boundary conditions are permeameter-like conditions: no flux across the lateral boundaries, constant head at upstream and downstream boundaries. The flow equation is solved numerically with a finite difference scheme [Ababou et al., 1989] (Figure 1b) using a grid of 1024 elements by 256 elements.

[7] Once the flow field is obtained, solute transport is simulated by a particle tracking method. Particle displacements are achieved at discrete time intervals [Kinzelbach, 1988] using a bilinear interpolation of the velocity field [Pollock, 1988]. Transport is purely advective and consequently, dispersion arises from particle spreading induced by the permeability field heterogeneity only. The influence of additional diffusion and local dispersion (i.e., dispersion within each of the element volumes) will be investigated in a subsequent work. Preliminary tests show that the optimal time step $\delta t$ is defined by the relation $\delta t=\frac{l_{\text {block }}}{10 v_{\max }}$ where $v_{\max }$ is the local block maximum velocity and $l_{\text {block }}$ is the characteristic block size. To present the results, we normalize particle travel distances by the log permeability field correlation length $\lambda_{\operatorname{lnK}}$ and the traveltime by a characteristic normalization time that we define as $\tau=\frac{n \lambda_{\ln K}}{\exp (\langle\ln K\rangle)|\nabla h|}$. In the simulations, we take the following parameters: the porosity $n=1$, the average $\log$ permeability $\exp (\langle\ln K\rangle)=1$ and the mean head gradient $|\nabla h|=1$. The correlation length of the $\log$ permeability field was 8 elements [Salandin and Fiorotto, 1998] so that the study area is $128 \lambda_{\operatorname{lnK}} \times 32 \lambda_{\operatorname{lnK}}$ (Figure 1). In order to account for the effect of the nonstationary zones near the domain boundaries, particles were injected 8 correlation lengths away from the side and upstream boundaries [Salandin and Fiorotto, 1998]. In the following, the symbol $v$ denotes the norm of the velocity vector normalized by the expected mean Eulerian velocity $\exp (\langle\ln K\rangle)|\nabla h| / n$.

\section{Conditional Distributions}

[8] To investigate the Lagrangian velocity field correlation structure, we study the distribution of velocities along the particle paths, conditionned by the particles initial velocity. The conditional velocity distribution is initially centered on the initial velocity $v_{0}$ and converges at late traveltime or travel distance toward the asymptotic velocity distribution. The rate of convergence toward the asymptotic distribution (in time or in distance) depends on the correlation structure of the velocity field. In this section, we first review the expected conditional distributions for multinormal random fields, which are classically used to model Eulerian velocity distributions in porous media [Rubin, 1990; Bellin et al., 1992]. Then, we describe the characteristics of the velocity distributions that we obtain for the simulated velocity fields in heterogeneous permeability fields.

\subsection{Expected Conditional Distributions for Multinormal Fields}

[9] We consider a multinormal random field $\mathrm{Y}$, defined by its mean $E(Y)$, its variance $\sigma$ and a correlation function $\rho(x)$, where $x$ is the lag distance between two points. For such random field, the probability of occurrence $P\left(y, x \mid y_{0}\right)$ of a value $y$ at position $x$, knowing its initial value $y_{0}$, is given by

$$
\begin{aligned}
P\left(y, x \mid y_{0}\right)= & \frac{1}{\sqrt{2 \pi \sigma^{2}\left(1-\rho^{2}(x)\right)}} \\
& \exp \left[-1 / 2\left(\frac{y-E(Y)-\rho(x)\left(y_{0}-E(Y)\right)}{\sigma \sqrt{1-\rho^{2}(x)}}\right)^{2}\right]
\end{aligned}
$$

where $\rho(x)$ is the random field correlation function and $E(Y)$ and $\sigma^{2}$ are respectively the random field mean and variance. Note that the random variable $y$ denotes any value of a multinormal random field (e.g., permeability or velocity field). 
a. log permeability field

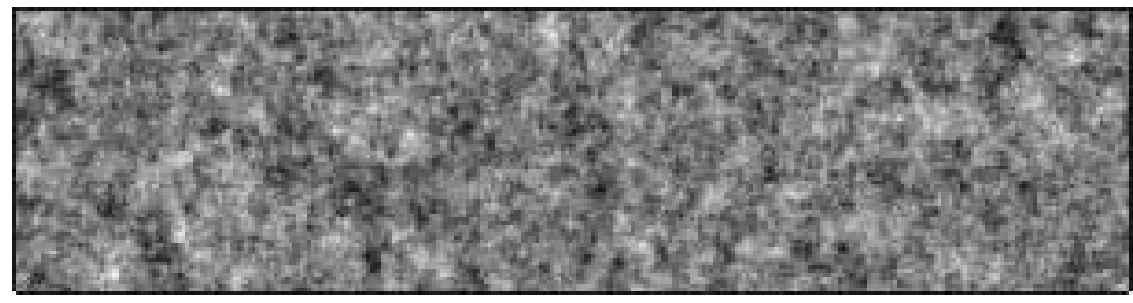

b. log velocity field for a permeability field variance of 0.25

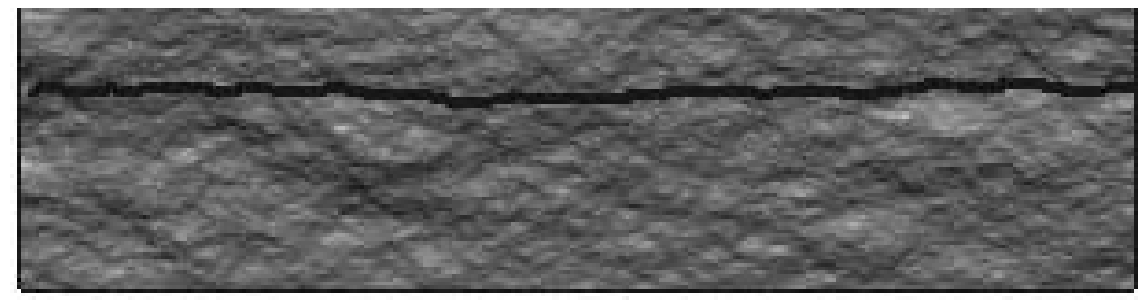

c. velocity distribution along the particle path

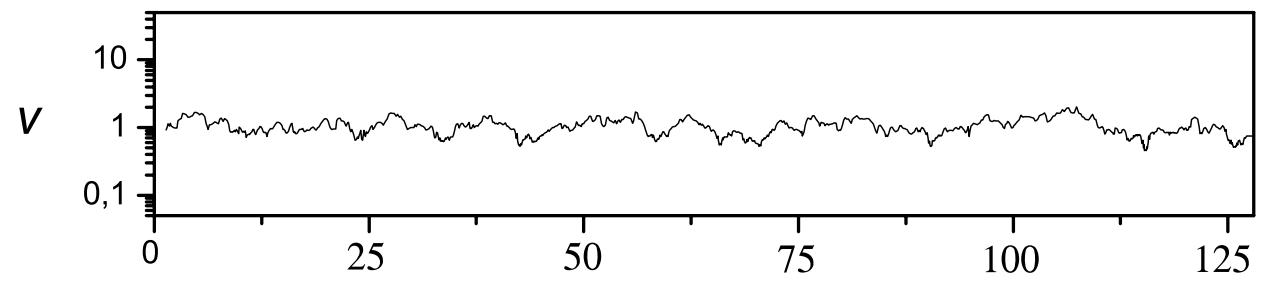

d. log velocity field for a permeability field variance of 3

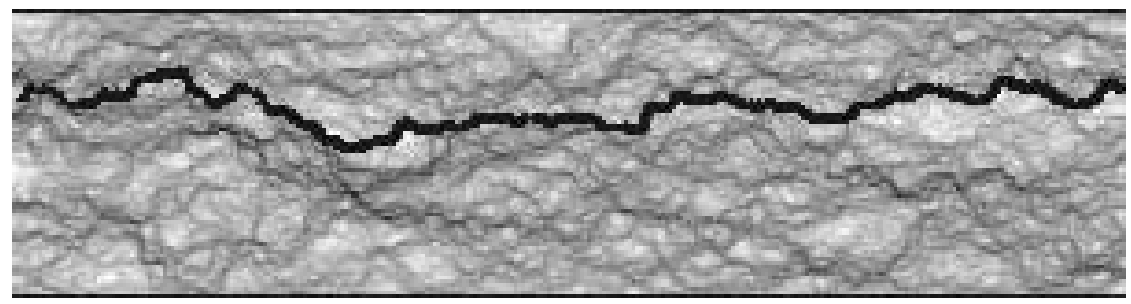

e. velocity distribution along the particle path

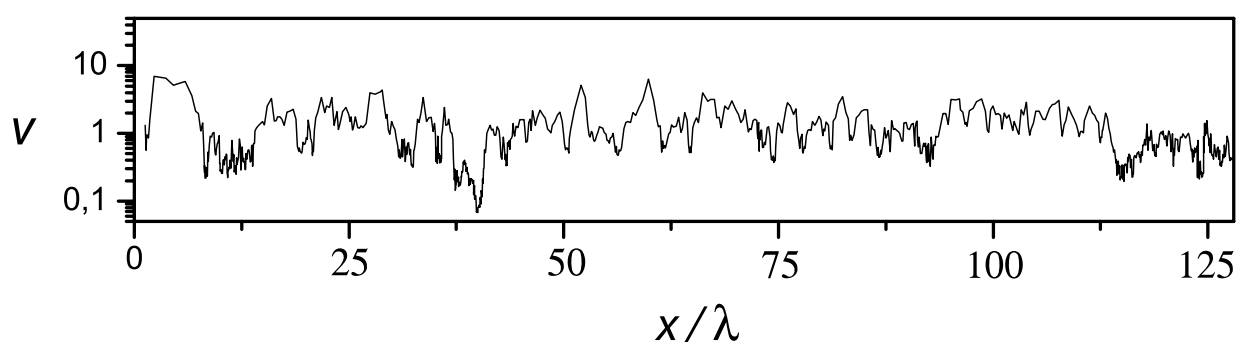

Figure 1. Example of (a) generated log permeability field realization for a $128 \lambda \times 32 \lambda$ grid with $\lambda_{\operatorname{lnK}}=8$, (b) calculated velocity field and example of a particle path for $\sigma^{2}{ }_{\operatorname{lnK}}=0.25$, (c) velocity sampled by the particle along the trajectory, (d) calculated velocity field and example of a particle path for $\sigma^{2}{ }_{\operatorname{lnK}}=3$, and (e) velocity sampled by the particle along the trajectory. The log permeability and log velocity amplitudes are represented on a color scale ranging from white for small values to black for large values. 


\section{a. example of conditional velocity distributions at different lag distances}

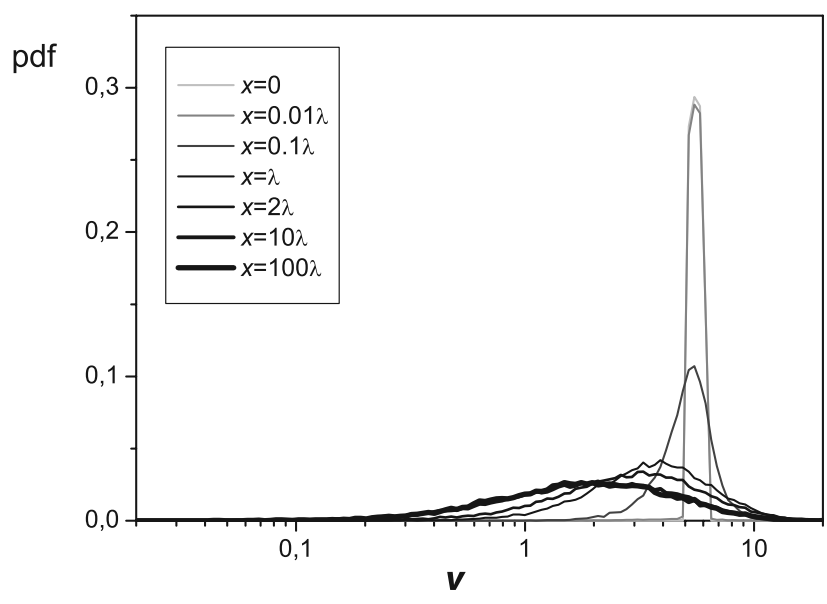

b. example of conditional velocity distributions at different lag times

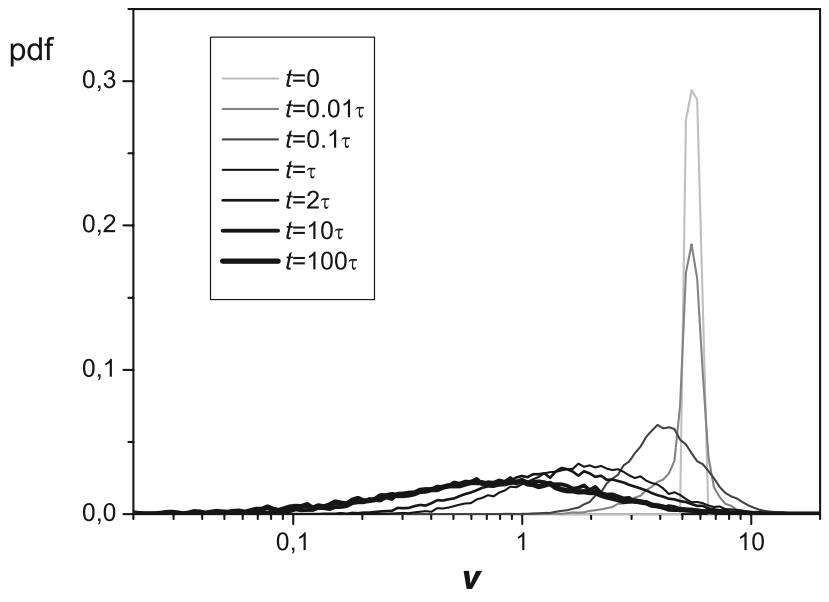

Figure 2. Example of conditional distributions for an initial velocity $v_{0}=6$ and a $\log$ permeability field variance $\sigma_{\operatorname{lnK}}^{2}=3$ (a) as a function of travel distance and (b) as a function of traveltime.

[10] Hence, for a multinormal field $Y$, the conditional distributions are expected to be normally distributed and are fully characterized by their conditional mean and variance that are given by

$$
\begin{gathered}
E\left(y, x \mid y_{0}\right)=E(y)+\rho(x)\left(y_{0}-E(y)\right) \\
\sigma^{2}\left(y, x \mid y_{0}\right)=\sigma^{2}(y)\left(1-\rho^{2}(x)\right)
\end{gathered}
$$

respectively.

[11] The evolutions of the conditional mean and variance with the lag distance $x$ are both characterized by the correlation function $\rho(x)$, which is independent of the initial value $y_{0}$ for a multinormal random field [Gomez-Hernandez and Wen, 1998]. In the following, we show that for a multinormal log permeability field, the velocity field deviates from the multinormal model characterized by equations (1)-(3).

\subsection{Conditional Lagrangian Velocity Distributions Obtained by Numerical Simulations}

[12] We analyze the conditional Lagrangian distributions of the absolute value of the flow velocity obtained from numerical simulations for log permeability field variances ranging from 0.25 to 3 . The conditional velocity distributions can be represented as a function of particle traveltime or travel distance (Figure 2). Velocity classes are defined based on a regular discretization of the log velocity field. An equal number of particles is injected in each velocity class. The obtained conditional velocity distributions are normalized by the number of injected particles in each class. As long as enough particles are injected in each velocity class to obtain reliable statistics, the method of injection does not influence the analysis of the conditional velocity distributions. Results are averaged over 500 realizations with 30000 particles departing from each velocity class. Figure 2 shows examples of conditional velocity distributions. At short traveltimes and distances, the conditional distributions are narrow, while at late traveltime and distance, they converge to the asymptotic Lagrangian velocity distribution. The preasymptotic conditional distributions are found to be highly skewed. The asymptotic distribution is close to a lognormal distribution, as previously obtained from numerical simulations [Bellin et al., 1992].

[13] Figure 3 shows the evolution of the conditional mean and variance of the Lagrangian velocity field versus the particle traveltime, for different initial velocities and a $\log$ permeability field variance $\sigma_{\ln K}^{2}=3$. The evolutions of the conditional mean and variance are found to be very dependent on the initial velocity, contrary to the multinormal case (equations 2 and 3). The convergence of the conditional mean to the asymptotic mean is longer for low initial velocities than for high initial velocities (Figure 3a). Furthermore, the increase toward the asymptotic variance is not steady like in the multinormal case (Figure 3b). For the lowest initial velocities ( $\ln v_{0}<-1.5$ ), the conditional variance increases above the asymptotic variance at intermediate time. Such effect is related to the skewness of conditional distributions which is visible even in the logscale representation (Figure 2). The persistence of a significant proportion of particles having velocities close to the initial velocity, while other particles have experienced the whole velocity field distribution, implies that the preasymptotic variance may be higher than the asymptotic variance.

[14] In summary, not only are the simulated velocity distributions not Gaussian, but their correlation structure differs from the multinormal representation, since the evolution of conditional distributions with traveltime or distance depend strongly on the initial conditioning velocity. This may explain the inconsistency of the Fickian representation for modeling dispersion in such velocity fields. In the following, we develop a method to quantify such nonmultinormal correlation structure and discuss how to represent it in effective transport models.

\section{Definition of Conditional Correlation Times and Lengths Estimates}

[15] The analysis of conditional velocity distributions shows that the convergence to the asymptotic velocity distribution depends on the initial velocity (Figure 3). In 

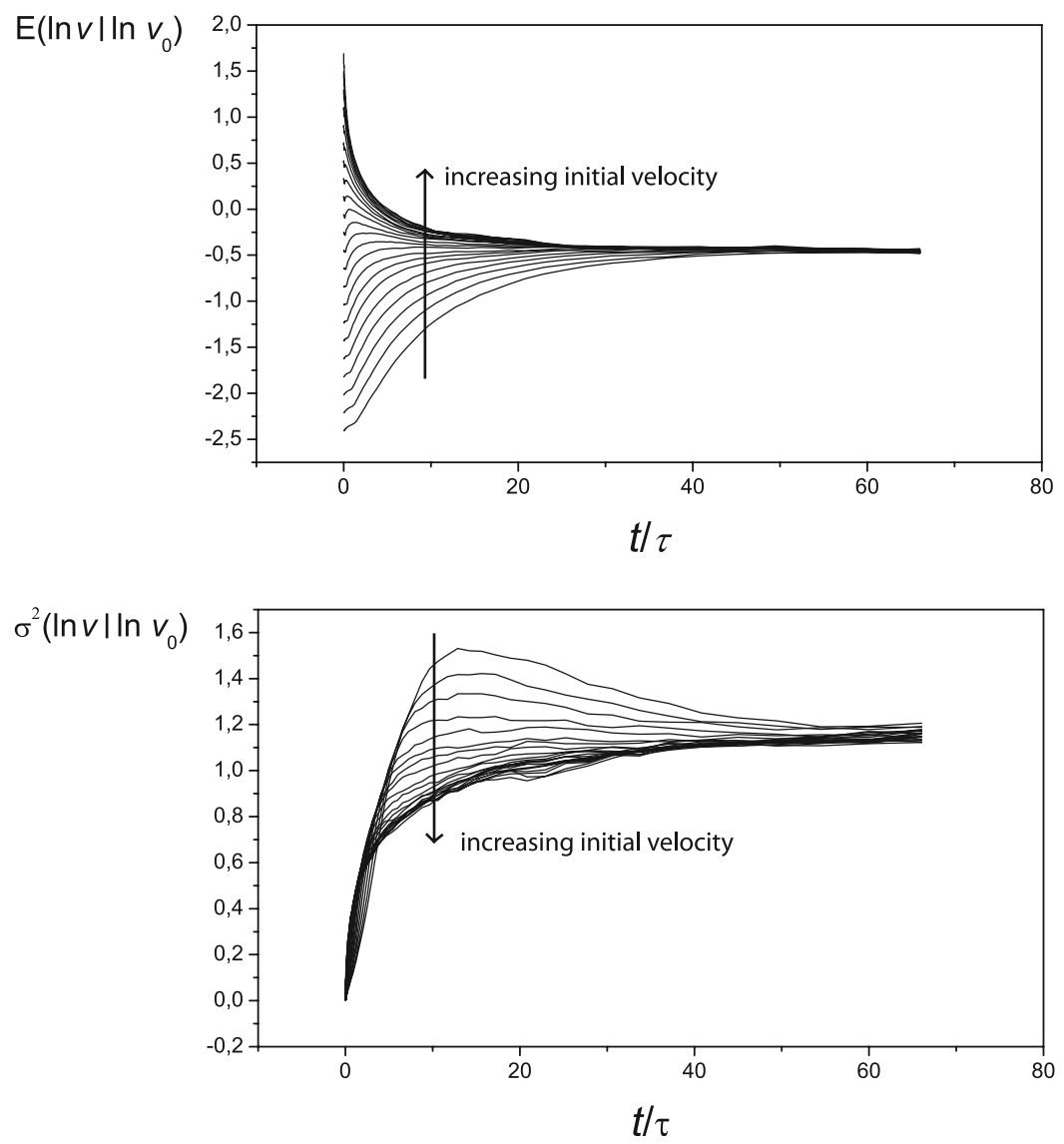

Figure 3. Evolution as a function of particle traveltime of the conditional mean and variance for a log permeability field variance $\sigma^{2}{ }_{\mathrm{lnK}}=3$, for different initial velocities $v_{0}$, with $0.1<v_{0}<6$.

this section, we discuss how to quantify this effect in terms of conditional correlation. A possible estimate of the conditional correlation is the conditional covariance. The conditional covariance $\operatorname{Cov}\left(y, y_{0}\right)$ is directly related to the conditional mean $E\left(y \mid y_{0}\right)$ by

$$
\begin{aligned}
\operatorname{Cov}\left(y, y_{0}\right) & =E\left(\left(y_{0}-E(y)\right)(y-E(y)) \mid y_{0}\right) \\
& =\left(y_{0}-E(y)\right)\left(E\left(y \mid y_{0}\right)-E(y)\right)
\end{aligned}
$$

where $y$ is the value of the random field at a given lag distance or lag time of the initial value $y_{0}$.

[16] The main problem with the use of the conditional covariance as an estimator of conditional correlation is its poor sensitivity for initial values close to the asymptotic mean. For initial velocities close to the mean $\left(-0.75<\ln v_{0}<\right.$ 0.25 ), the conditional mean curves, which are linearly related to the conditional covariances (equation 4), overshoot the asymptotic value before converging back toward it (Figure 3a). This may be explained by the fact that particles initially sample preferentially high velocities, since a long time is required to sample the lowermost portion of the velocity distribution. Hence the use of conditional covariance to quantify conditional correlation leads to inconsistent results.

[17] Conversely, a relevant estimate of the conditional correlation could be provided by the conditional variance (Figure 3b). It expresses the rate at which the velocity variability increases with distance or time interval. However, for the velocity fields considered here, the increase toward the asymptotic variance is not steady like in the multinormal case (Figure 3b). Again, this effect disqualifies the use of the conditional variance as an estimator of the conditional correlation. In summary, the use of the conditional covariance or conditional variance for estimating correlation is valid for multi-Gaussian models, for which the conditional distributions are fully described by their mean and variance (equations (1), (2), and (3)), but not in the present case, for which conditional distributions are skewed.

[18] An estimator of the correlation time and length valid for any type of velocity distributions is required. We define the correlation time and length as generic characteristics that quantify the convergence to the asymptotic regime. To determine the rate of convergence toward the Lagrangian velocity distribution, we calculate the mean absolute difference between the conditional distributions and the asymptotic Lagrangian velocity distribution:

$$
C\left(x \mid \ln v_{0}\right)=\int_{-\infty}^{\infty}\left|P\left(\ln v \mid \ln v_{0}, x\right)-P(\ln v, \infty)\right| d \ln v
$$

where $x$ represents either the lag distance or the lag time, $P\left(\ln v \mid \ln v_{0}, x\right)$ is the conditional $\log$ velocity distribution and $P(\ln v, \infty)$ is the asymptotic Lagrangian log velocity distribution. The asymptotic Lagrangian distribution is computed as the late traveltime or the large travel distance 
a. difference between the conditional distributions and the asymptotic distribution as a function of lag distance

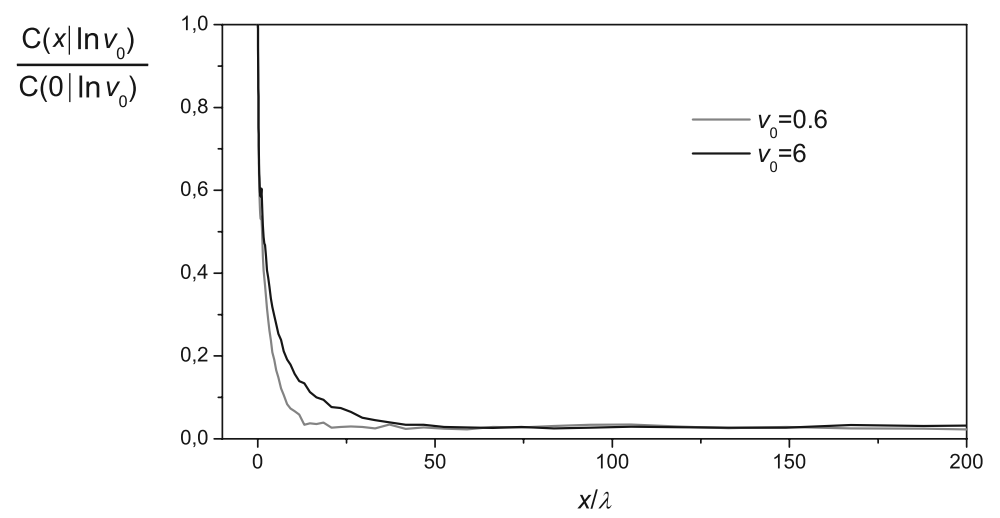

b. difference between the conditional distributions and the asymptotic distribution as a function of lag time

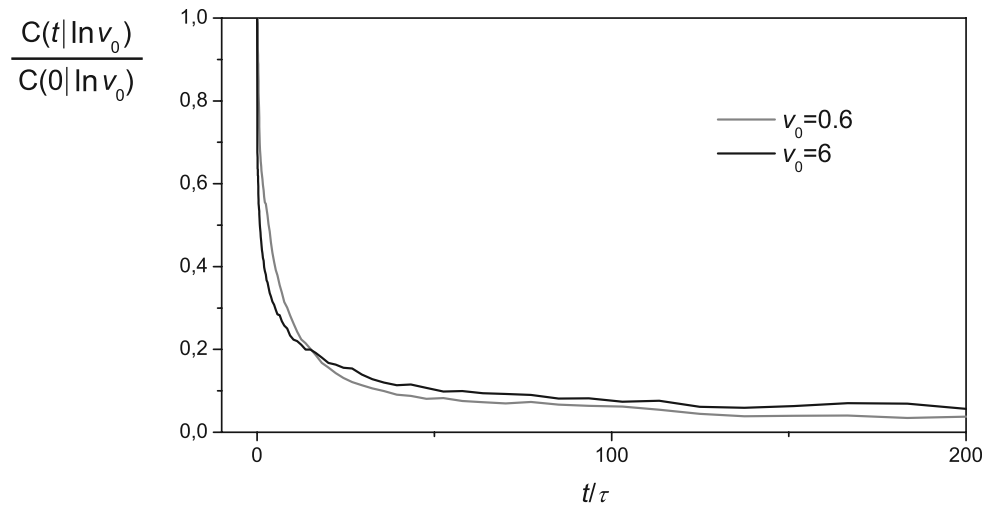

Figure 4. Evolution of the difference between the conditional distributions and the asymptotic distributions (equation (5)) for a $\log$ permeability field variance $\sigma_{\operatorname{lnK}}^{2}=3$ (a) as a function of travel distance and (b) as a function of traveltime. The curves are normalized by their value for $t=0$ or $x=0$.

velocity distribution of particles departing from all the possible initial velocities. In practice, the asymptotic Lagrangian distributions were reached by $\mathrm{x}=100 \lambda$ and $\mathrm{t}=100 \tau$, therefore the distributions for that distance and time (Figure 2) are approximately identical to the asymptotic distribution.

[19] All distributions are shifted by the conditional mean (i.e., to be centered on the same mean) in order to avoid side effects related to the shape of the distributions without loosing information on the velocity correlation. When $C\left(x \mid \ln v_{0}\right)=0$, the conditional velocity distribution is equal to the asymptotic distribution and particles have lost memory of there initial velocity $v_{0}$. We estimated the characteristic correlation lengths or time by integrating this function in distance and time respectively. In order to filter out the marginal differences between the distributions at late time or distance, space and time integrations are performed until the difference between the conditional distributions and the asymptotic distribution (equation 5) is lower than 0.05 . Preliminary tests showed that stopping when the residual is less than $5 \%$ allowed avoiding integrating the numerical variability, without loosing information on the velocity correlation. Hence the characteristic correlation length is obtained by integrating the correlation function over distance:

$$
x_{C}\left(\ln v_{0}\right)=\int_{0}^{x_{\max }} C\left(x \mid \ln v_{0}\right) / C\left(0 \mid \ln v_{0}\right) d x
$$

where $\mathrm{x}$ represents either travel distance or time and $x_{\max }$ represents the maximum distance of integration. The characteristic correlation time $t_{\mathrm{c}}$ is obtained similarly by integrating the correlation function over time.

[20] We tested this method from particle tracking simulations for two end-members of possible velocity field structures: a multinormal velocity field (defined by equation (1)) and a stratified velocity field. In the first case, the correlation distance is expected to be independent on the initial velocity while in the second case the correlation distance is expected to be strongly dependent on the initial velocity. For a multinormal random field defined by equation (1), we obtain a consistent estimate of the conditional correlation, independent of the initial conditioning value, as expected for multinormal random fields. The integral $x_{\mathrm{c}}$ is not strictly equal to the multinormal field 
a. characteristic length as a function of initial velociy

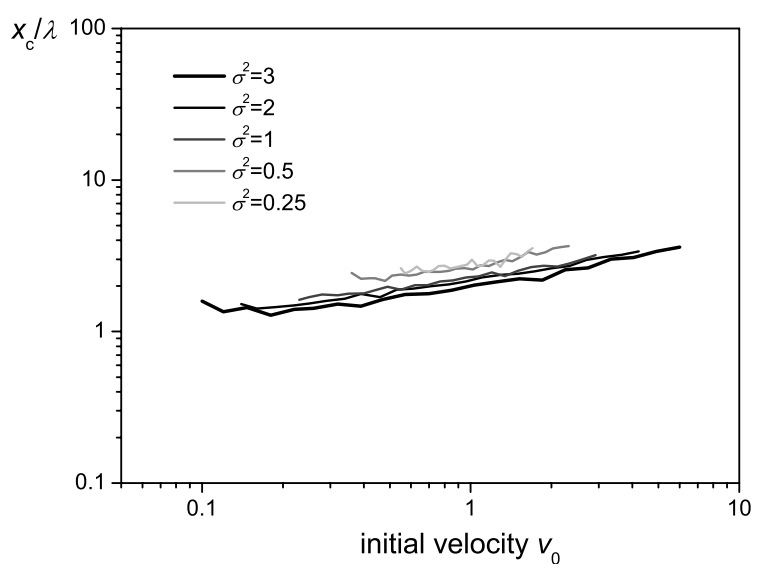

b. characteristic time as a function of initial velociy

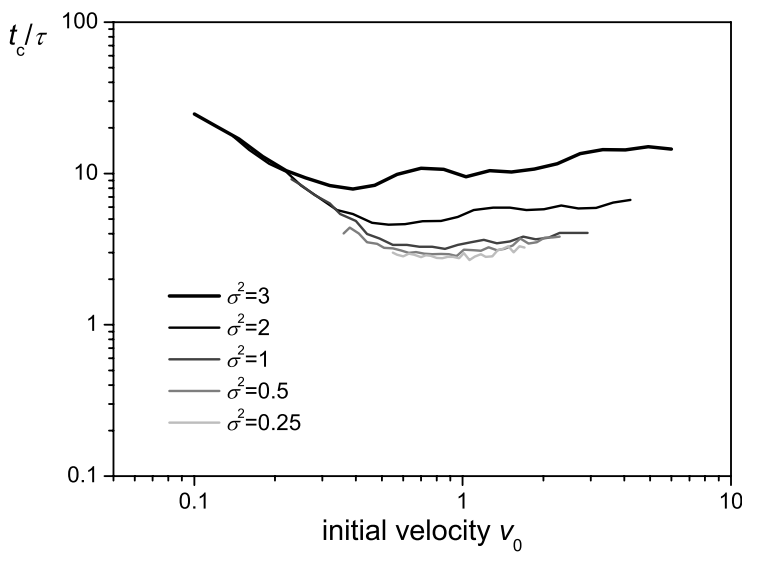

Figure 5. (a) Correlation distance as a function of conditioning initial velocity and (b) correlation time as a function of conditioning initial velocity for different log permeability field variances. The estimates of correlation time and length are calculated as the integral of the correlation curves in Figure 4 (equation (6)).

correlation length $\lambda$ but it is proportional. $x_{\mathrm{c}}$ is well related to the correlation length: whatever the multinormal field correlation length and variance are, we obtain: $x_{\mathrm{c}}=K \lambda$, with the coefficient $K=0.185 \pm 0.002$. For numerical simulations of solute transport in stratified velocity fields with lateral mass exchange by diffusion [Matheron and de Marsily, 1980], we obtain velocity correlation functions in time that are independent of the initial particle velocity, while the velocity correlation functions in distance depend on the conditioning initial particle velocity. In such media, for particles initially departing from a velocity $v_{0}$, the probability of sampling the velocity $v_{0}$ at time $t$ is decreasing with a power law defined by $P(t) \propto t^{-0.5}$ [Bouchaud and Georges, 1990]. As expected, the obtained temporal correlation functions decrease with a power in the range $-0.5 \pm 0.05$, whatever the initial velocity $v_{0}$.

[21] In summary, the method proposed to characterize the conditional correlation (equation (5)) can be used for highly skewed velocity distributions and gives consistent results for end-members of possible velocity field correlation structures. In the next section, we apply this method to velocity fields simulated in mutlinormal log permeabiliy fields.

\section{Results: Lagrangian Velocity Field Correlation Structure in Multinormal Log Permeability Fields}

[22] Figure 4 shows the evolution of the conditional Lagrangian velocity correlation $\mathrm{C}\left(x \mid \ln v_{0}\right) / \mathrm{C}\left(0 \mid \ln v_{0}\right)$ as a function of particle traveltime and travel distance for a $\log$ permeability field of variance $\sigma_{\operatorname{lnK}}^{2}=3$ for two different initial velocities. These curves display the rate of convergence of conditional distributions toward the asymptotic Lagrangian velocity distribution. The shapes of the curve are different for low and high initial velocity, which suggests that the conditional velocity correlation depends on the initial velocity. To quantify the conditional correlation, we compute the integral of these functions that gives the characteristic convergence time or distance (equation (6) and Figure 5).

\subsection{Spatial Conditional Correlation}

[23] The results for the correlation in distance (Figure 5a) indicate that the distance for convergence to the Lagrangian distribution increases with velocity. Such result implies that particles loose memory of their initial velocity over shorter distances for low initial velocities than for high initial velocities. This could be related to the different spatial organization of low- and high-velocity zones. Indeed, for large log permeability variances, low- and high-velocity zones do not have the same spatial organization (Figure 1). High-velocity zones form tortuous channels, whereas lowvelocity values are localized in island shaped zones. The longitudinal size of the high-velocity channels is larger than that of the low-velocity islands between the channels. Results show that the correlation length $x_{\mathrm{c}}$ scales with velocity as a power law:

$$
x_{\mathrm{c}}=A\left(\sigma^{2}\right) v_{0}^{m}
$$

with $m=0.3 \pm 0.02$.

\subsection{Temporal Conditional Correlation}

[24] For the temporal conditional correlation, we observe that the evolution of the conditional distributions differs depending on whether the initial velocity is low or high (Figure 4b). The correlation curves conditioned on high initial velocities decrease rapidly initially and then converge slowly at late time. Consequently, it takes a long time for particles departing from high velocities to sample the whole velocity distribution and especially the low velocities. Without diffusion, mass conservation implies that particles traveling from high to low velocities experience a range of intermediate velocities. Since particles cannot jump directly from high to low velocities, the highest the initial velocity, the longer it takes to sample the lowest velocities. On the other hand, the correlation curves conditioned on low initial velocities decrease initially slowly but drop to zero faster. It 


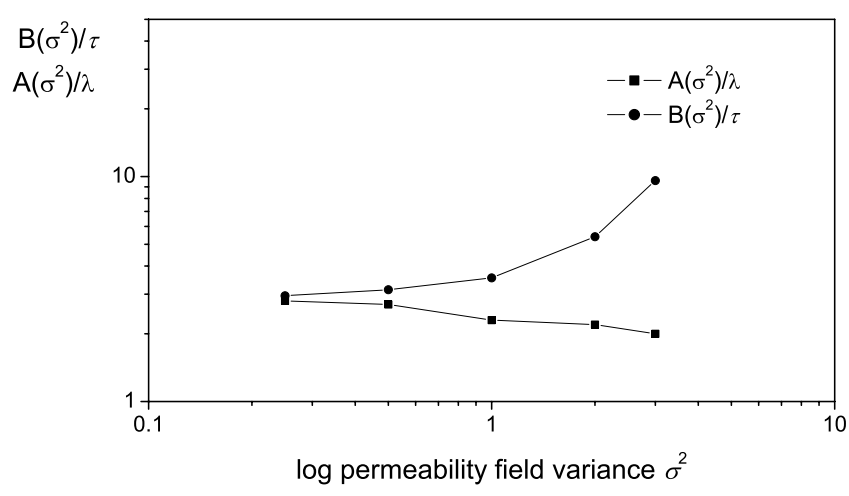

Figure 6. Evolution as a function of the $\log$ permeability field variance of the coefficients $A$ and $B$ that characterize the evolution of the correlation times and distances with $v_{0}$ (equations (7) and (8)).

takes a long time for particles to get out of low-velocity zones, but when they are out eventually, they sample rapidly the whole velocity field.

[25] The balance between these opposite effects produces two distinctly different correlation regimes. For the lowermost part of the velocity distribution, transport is controlled by the time needed for particles to leave lowvelocity zones. The dependence of the correlation time in this regime is $t_{\mathrm{c}}=2 \lambda / v_{0}$. Consequently, the characteristic correlation time is the same as if particles would have to leave a region of size $2 \lambda$ at constant initial velocity $v_{0}$. For intermediate and high initial velocities, the time for particles to sample the whole velocity distribution becomes higher than the time needed for particles to leave the initial velocity. The evolution of correlation time $t_{\mathrm{c}}$ with the initial velocity is a slow increase following a power law:

$$
t_{\mathrm{c}}=B\left(\sigma^{2}\right) v_{0}^{n}
$$

with $n=0.2 \pm 0.02$.

[26] We find that the relation between the conditional correlation time and length is not given by $v_{0}=x_{\mathrm{c}} / t_{\mathrm{c}}$. This is due to the fact that in the preasymptotic regime $\left(t<t_{\mathrm{c}}\right.$ and $x<x_{\mathrm{c}}$ ), the particle velocity is not necessarily equal to the initial velocity $v_{0}$. Its probability distribution is given by the conditional velocity distributions (Figure 2).

\section{Discussion}

\subsection{Influence of the Permeability Field Heterogeneity on the Velocity Field Organization}

[27] For the different log permeability field variances in the range $0.25-3$, the correlation length curves as a function of initial velocity are approximately superimposed and characterized by the same power law exponent. Although the velocity channeling is apparently increasing with the $\log$ permeability field variance (Figure 1), we do not observe a very important effect on the Lagrangian velocity correlation lengths. the scaling of the correlation length as a function of the initial velocity is independent on the variance (Figure 5a). Increasing the permeability field variance causes the asymptotic Lagrangian velocity distribution to shift toward the higher velocities but it does not change the velocity field correlation structure. Similarly, the scaling of the correlation time as a function of the initial velocity is independent on the permeability field variance. However, the prefactor B (equation (8)) does depend on the permeability field variance.

[28] Figure 6 displays the coefficients $A$ and $B$ that characterize the evolution of the correlation times and distances with $v_{0}$ (equations (7) and (8)) as a function of the $\log$ permeability field variance. The evolution of $A$ is a slow decrease with $\sigma_{\operatorname{lnK}}^{2}$. A similar decrease of the correlation length with the log permeability field variance was obtained by Salandin and Fiorotto [1998], who analyzed the Eulerian velocity field covariance of lognormal permeability fields. Conversely, $B$ increases rapidly with the $\log$ permeability field variance. Therefore the main effect of increasing the log permeability variance is to increase the velocity field correlation time and not the correlation length. This increase is similar to the increase in the convergence time to Fickian dispersion as the log permeability variance increases and to the increase in the asymptotic dispersion coefficient as the log permeability variance increases [Salandin and Fiorotto, 1998].

\subsection{Consequences for the Influence of the Injection Method}

[29] The first consequence of the results obtained is concerning the influence of the injection method on solute dispersion. When the initial solute velocity distribution is different from the asymptotic Lagrangian velocity distribution, there is a non stationary regime, in which the Lagrangian velocity distribution is evolving from the initial velocity distribution to the asymptotic velocity distribution. For instance in tracer tests, the tracer may be injected in a low-velocity zone compared to the distribution of velocities in the media. In such case, the distribution of velocities seen by the tracer would evolve as a function of traveltime or distance untill it reaches an asymptotic velocity distribution. Our results suggest that when the tracer is in the preasymptotic regime, velocity distributions are highly skewed, with a large portion of the mass moving with the initial velocity and a small portion of the mass having very different velocities (Figure 2). Hence, in this regime, transport would be expected to have important non-Fickian characteristics. In practical applications, this non stationary regime may be relatively long and dominate the characteristics of the tracer dispersion.

[30] The duration and the distance over which the non stationary regime is expected to occur should depend on the velocity in which the tracer was injected. For instance, injecting the solute preferentially in high-velocity zones implies that convergence toward the asymptotic regime will be initially quick and then slow at late time. Indeed, we showed that it takes a long time for particles departing from high velocities to sample the whole velocity distribution and especially the low velocities (Figure $4 \mathrm{~b}$ ). The average distance to reach the asymptotic regime would be larger if particles are preferentially injected in high-velocity zones than in low-velocity zones (Figure 5a). 


\subsection{Consequences for the Representation of the Velocity Field Organization in Upscaled Transport Models}

[31] The results of the present study have important consequences for the representation of velocity transitions along particle paths in upscaled transport models. Continuous time random walks (CTRW) models are increasingly used in subsurface hydrology to account for the anomalous spreading of tracer, that exhibit skewness or heavy power law tails [Berkowitz et al., 2002; Dentz et al., 2004]. The CTRW representation assumes that the velocity transitions along the particle paths can be represented by random particle jumps performed in random times, which correspond to random velocities. The jumps sizes and times are independent on the previous jump sizes and times. Thus the particle jumps correspond to the velocity correlation length and the jump times, or waiting times, to the velocity correlation time. The analytical solutions are often obtained by assuming a Markovian assumption in space or in time, which correspond respectively to a constant jump size or jump time. Hence these solutions implicitly assume particular values for the exponents $m$ and $n$ (respectively $m=0$ or $n=0$ in equations (7) and (8)). Comparisons with numerical simulations, lab and field experiments showed that, in several cases, the Markovian in space simplification works well [Berkowitz et al., 2006]. The present study suggests that, for the particular permeability fields investigated, the particle waiting times and the subsequent jumps are not independent, since the characteristic correlation time and distance depend on the particle velocity. Such property can be implemented in CTRW formulations where spatial and temporal increments are coupled [Shlesinger et al., 1982; Blumen et al., 1989; Berkowitz and Scher, 1998; Meerschaert et al., 2003; Metzler and Klafter, 2000, 2004]. Such theoretical framework could be used to account for a variety of possible relations between conditional correlation and velocity, including non linear relationships, such as obtained in our study (equations (7) and (8)).

[32] Obviously, the results presented in this study are relevant to multinormal $\log$ permeability field defined by an exponential correlation function and advection transport only. We expect that other types of Lagrangian velocity correlation structures may be obtained in other cases. The characteristic exponent of the velocity correlation structure (equations 7 and 8) appear to be quite general since they are independent on the log permeability field variance. It is probable that they are relevant for multinormal log permeability field with a finite covariance. In three dimensions, the channeling of high-velocity zones may be even more important than in two dimensions, as flow has more opportunity to encounter least resistance preferential flow paths. An increased channeling is also expected to occur in nonmultinormal permeability fields with preferentially connected high-permeability zones [Zinn and Harvey, 2003]. In the general case, the role of channeling on the velocity field structure is not obvious. In the present study, we found that the apparent increase in channeling for highpermeability field variances does not imply an increased correlation length of the high-velocity zones. It implies an increase of the low-velocity zones correlation time, as the asymptotic velocity distribution is shifted toward the high velocities. These aspects will be investigated in further studies. The role of diffusion and local dispersion, which are likely to change significantly the velocity transition properties along the particles paths, will also be investigated in the future.

\section{Conclusions}

[33] The present study shows that the correlation structure of the Lagrangian velocity field in log permeability fields is complex and may not be simply described by classical statistical methods. How to quantify non-multi-Gaussian correlation structures is a debated issue [Gomez-Hernandez and Wen, 1998; Knudby and Carrera, 2005]. Here we tackle this issue by studying the evolution of Lagrangian velocity distributions conditioned on the initial particle velocity as a function of particle traveltime or distance. We showed that, in multinormal log permeability fields, the Lagrangian velocity field is not multinormal, since the conditional Lagrangian velocity distributions depend on the initial particle velocity.

[34] The conditional velocity distributions, which are initially narrow and centered on the initial velocity, widen as particles experience different velocities, and converge to the asymptotic Lagrangian velocity distribution. The rate of convergence toward the asymptotic Lagrangian velocity distribution depends on the velocity correlation function. To quantify the rate of convergence as a function of initial velocity, we computed the mean absolute difference between conditional distributions and the asymptotic Lagrangian distribution at different particle traveltimes and travel distances. We thus defined estimates of correlation length and time conditioned on initial particle velocities in a Lagrangian framework. These Lagrangian velocity field correlation length and time quantify the traveltime and distance above which a particle velocity is independent of its initial velocity.

[35] For the correlation in distance, the evolution with the initial particle velocity $v_{0}$ is an increase characterized by a power law exponent of $0.3: x_{\mathrm{c}}=A\left(\sigma^{2}\right) v_{0}^{0.3}$. For the correlation in time, the evolution with initial particle velocity is not monotonic. For low initial velocity, the dominant effect is the time needed for particles to leave the low-velocity zones and the correlation is inversely proportional to the initial velocity: $t_{\mathrm{c}}=2 \lambda / v_{0}$. For intermediate and high velocities, the time for particle to sample the whole velocity distribution becomes higher than the time needed for particles to leave the initial velocity and the correlation time increases with initial velocity: $t_{\mathrm{c}}=B\left(\sigma^{2}\right) v_{0}^{0.2}$.

[36] The scaling of the correlation length and time as a function of the initial velocity $v_{0}$ is independent on the log permeability field variance. The main effect of increasing the log permeability variance is to increase the coefficient $B\left(\sigma^{2}\right)$, while the coefficient $A\left(\sigma^{2}\right)$ is slightly decreasing. Hence the increase in flow channeling that is observed for high log permeability variances induces mainly an increase of the velocity field correlation time. The nonlinear dependence of both the correlation length and the correlation time on the particle initial velocity restricts the use of spatial or temporal Markovian assumptions for modeling particle velocity transitions in heterogeneous media. This property can be accounted for in coupled CTRW formulations. 
[37] Acknowledgments. We thank Katia Besnard for sharing her numerical code for simulating solute transport in heterogeneous permeability fields. Philippe Gouze is thanked for his helpful comments on the manuscript. This work was partly financed by the European project "SALTRANS" (contract EVK1-CT-2000-00062) and by the city of Plomeur. We also wish to thank the French CNRS for their financial support, in particular through an ATIP project and through the INSUE project "ORE $\mathrm{H}+$ ".

\section{References}

Ababou, R., D. McLaughin, L. W. Gelhar, and A. F. B. Tompson (1989), Numerical simulation of three-dimensional saturated flow in randomly heterogeneous porous media, Transp. Porous Media, 4, 549-565.

Adams, A. E., and L. W. Gelhar (1992), Field study of dispersion in a heterogeneous aquifer: 2. Spatial moments analysis, Water Resour. Res., 28, 3293-3307.

Becker, M. W., and A. M. Shapiro (2003), Interpreting tracer breakthrough tailing from different forced-gradient tracer experiment configurations in fractured bedrock, Water Resour. Res., 39(1), 1024, doi:10.1029 2001WR001190.

Bellin, A., P. Salandin, and A. Rinaldo (1992), Simulation of dispersion in heterogeneous porous formations: Statistics, first-order theories, and convergence of computations, Water Resour. Res., 28, 2211-2277.

Benson, D. A., S. W. Wheatcraft, and M. M. Meerschaert (2000), Application of a fractional advection-dispersion equation, Water Resour. Res., 36, $1403-1412$.

Berkowitz, B., and H. Scher (1998), Theory of anomalous chemical transport in random fracture networks, Phys. Rev. E, 57, 5858-5869.

Berkowitz, B., J. Klafter, R. Metzler, and H. Scher (2002), Physical pictures of transport in heterogeneous media: Advection-dispersion, randomwalk, and fractional derivative formulations, Water Resour. Res., 38(10), 1191, doi:10.1029/2001WR001030.

Berkowitz, B., A. Cortis, M. Dentz, and H. Scher (2006), Modeling nonFickian transport in geological formations as a continuous time random walk, Rev. Geophys., 44, RG2003, doi:10.1029/2005RG000178.

Blumen, A., G. Zumofen, and J. Klafter (1989), Transport aspect in anomalous diffusion: Levy Walks, Phys. Rev. A, 40(7), 3964-3973.

Bouchaud, J.-P., and A. Georges (1990), Anomalous diffusion in disordered media: Statistical mechanism and physical applications, Phys. Rep., 195, 127-293.

Dagan, G. (1989), Flow and Transport in Porous Formations, Springer, New York.

Dentz, M., A. Cortis, H. Scher, and B. Berkowitz (2004), Time behavior of solute transport in heterogeneous media: Transition from anomalous to normal transport, Adv. Water Resour., 27, 155-173.

Gelhar, L. W. (1993), Stochastic Subsurface Hydrology, Prentice-Hall, Upper Saddle River, N. J.

Gomez-Hernandez, J. J., and X.-H. Wen (1998), To be or not to be mutliGaussian? A reflection on stochastic hydrogeology, Adv. Water Resour., $21,47-61$.

Haggerty, R. S., and S. M. Gorelick (1995), Multiple-rate mass transfer for modeling diffusion and surface reactions in media with pore-scale heterogeneity, Water Resour. Res., 31, 2383-2400.
Haggerty, R. S., S. A. McKenna, and L. C. Meigs (2000), On the late-time behavior of tracer test breakthrough curves, Water Resour. Res., 36, $3467-3479$.

Harvey, C. F., and S. M. Gorelick (2000), Rate-limited mass transfer or macrodispersion: Which dominates the plume evolution at the Macrodispersion Experiment (MADE) site?, Water Resour. Res., 36, 637-650.

Kinzelbach, W. (1988), The random walk method in pollutant transport simulation, in Ground Water Flow and Quality Modelling, edited by E. Custodio, A. Gurgui, and J. P. Lobo Ferreira, pp. 227-246, Springer, New York.

Knudby, C., and J. Carrera (2005), On the relationship between indicators of geostatistical, flow and transport connectivity, Adv. Water Resour., 28 , $405-421$.

Labolle, E. M., and G. E. Fogg (2001), Role of molecular diffusion in contaminant migration and recovery in an alluvial aquifer system, Transp. Porous Media, 42, 155-179.

Liu, G., C. Zheng, and S. M. Gorelick (2004), Limits of applicability of the advection-dispersion model in aquifers containing connected high-conductivity channels, Water Resour. Res., 40, W08308, doi:10.1029/ 2003WR002735

Matheron, G., and G. de Marsily (1980), Is transport in porous media always diffusive? A counterexample, Water Resour. Res., 16, 901-917.

Meerschaert, M. M., D. A. Benson, P. Becker-Kern, and H.-P. Scheffler (2003), Governing equations and solutions of anomalous random walk limits, Phys. Rev. E, 66, 060102 (R), doi:10.1103/PhysRevE.66.060102.

Metzler, R., and J. Klafter (2000), The random walk's guide to anomalous diffusion: A fractional dynamics approach, Phys. Rep., 339, 1-77.

Metzler, R., and J. Klafter (2004), The restaurant at the end of the random walk: Recent developments in the description of anomalous transport by fractional dynamics, J. Phys. A Math. Gen., 37, R161-R208.

Pollock, D. W. (1988), Semianalytical computation of path lines for finitedifference models, Ground Water, 26, 743-750.

Ruan, F., and D. McLaughin (1998), An efficient multivariate random field generator using the Fourier transform, Adv. Water Resour., 21, 385-399.

Rubin, Y. (1990), Stochastic modeling of macrodispersion in heterogeneous porous media, Water Resour. Res., 26, 133-141.

Salandin, P., and V. Fiorotto (1998), Solute transport in highly heterogeneous aquifers, Water Resour. Res., 34, 949-961.

Shlesinger, M. F., J. Klafter, and Y. M. Wong (1982), Random walks with infinite spatial and temporal moments, J. Stat. Phys., 27, 499-512.

Sudicky, E. A. (1986), A natural gradient experiment on solute transport in a sand aquifer: Spatial variability of hydraulic conductivity and its role in the dispersion process, Water Resour. Res., 22, 2069-2082.

Tsang, C.-F., and I. Neretnieks (1998), Flow channeling in heterogeneous fractured rocks, Rev. Geophys., 36, 257-298.

Zinn, B., and C. F. Harvey (2003), When good statistical models of aquifer heterogeneity go bad: A comparison of flow, dispersion, and mass transfer in connected and multivariate Gaussian hydraulic conductivity fields, Water Resour. Res., 39(3), 1051, doi:10.1029/2001WR001146.

O. Bour, P. Davy, J.-R. de Dreuzy, and T. Le Borgne, Géosciences Rennes, UMR 6118, CNRS, Université de Rennes 1, Campus de Beaulieu, F-35042 Rennes cedex, France. (leborgne@msem.univ-montp2.fr) 